DE

M E D I C I N A

T R O P I C A L

$\mathrm{DE}$

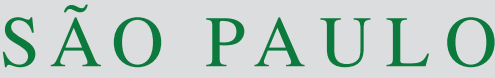

JOURNAL OF THE SÃO PAULO INSTITUTE OF TROPICAL MEDICINE

${ }^{1}$ Universidade Federal do Triângulo Mineiro, Departamento de Clínica Cirúrgica,

Uberaba, Minas Gerais, Brazil

2Universidade Federal do Triângulo Mineiro, Faculdade de Medicina, Uberaba, Minas

Gerais, Brazil

${ }^{3}$ Universidade Federal do Triângulo Mineiro, Departamento de Clínica Médica, Uberaba, Minas Gerais, Brazil

Correspondence to: Adilha Misson Rua Micheletti

Universidade Federal do Triângulo Mineiro, Departamento de Clínica Cirúrgica, Rua Getúlio Guaritá, 130, CEP 38025-440, Uberaba, MG, Brazil

Tel: +5534 3318-5152

E-mail: adilharua@yahoo.com.br

Received: 25 May 2019

Accepted: 30 October 2019

\section{Main findings and diagnostic yield of bronchoalveolar lavage, bronchial brushing and transbronchial biopsy in HIV-positive patients}

\author{
João Paulo Vieira dos Santos', Lucas Fabiano Garcia Leite ${ }^{2}$, Sheila Jorge \\ Adad$^{1}$, Mário-León Silva Vergara $^{3}$, Adilha Misson Rua Micheletti ${ }^{\circledR 1}$
}

\section{ABSTRACT}

Bronchoalveolar lavage, bronchial brushing and transbronchial biopsy are of fundamental importance in the diagnosis of pathologies affecting the lungs of immunosuppressed patients, especially those infected with HIV. This was a descriptive and retrospective study, in which the results of bronchoalveolar lavages, bronchial brushings and transbronchial biopsies of HIV-positive patients attended at the Clinical Hospital of the Federal University of Triangulo Mineiro from 1999 to 2015 were reviewed to determine the most frequent findings in these patients, to evaluate the diagnostic accuracy of these procedures and to correlate bronchoscopy results with clinical and radiological findings. Serological tests for HIV were confirmed and cases with negative or unverified serology were excluded. Medical records were reviewed for correlation with clinical and radiological findings. A total of 1,423 patients with a mean age of 50 years were initially selected; 727 cases had no serology for HIV; 696 had serology for HIV and 64 were positive. Of these, 47 were men, aged 24 to 84 years, and 17 women, aged 31 to 69 years. Biopsies and cytological tests were positive in 20 (31.25\%) of the 64 patients and the most frequent diagnosis was pneumocystosis, found in 8 cases $(12.5 \%)$. Of the 20 bronchofibroscopy-positive patients, only 2 did not show agreement between histopathological and clinical-radiological diagnoses. The analysis of the cytological tests and biopsy specimens obtained by bronchofibroscopy seems to be valuable for the etiological diagnosis of pulmonary infections in HIV- positive patients; however, negative results do not always exclude the diagnosis. In these cases, clinical symptoms and imaging findings may help to guide the best therapy.

KEYWORDS: HIV. AIDS. Bronchoscopy. Pneumocystis. Tuberculosis. Pulmonary infections. Lung infections. Bronchoalveolar lavage. Bronchial brushing. Transbronchial biopsy.

\section{INTRODUCTION}

The acquired immunodeficiency syndrome (AIDS) pandemic is characterized by superlative and frightening statistics. Since its beginning in the 1980s, 35 million people have been infected with the human immunodeficiency virus (HIV); approximately 37 million people around the world live with the virus and one person is infected with HIV every 17 seconds ${ }^{1}$.

HIV infection is characterized by a broad spectrum of clinical presentations from the acute phase to the advanced stages of the disease. In untreated individuals, the estimated average time between infection acquisition and disease onset is about 10 years. While the CD4+ T lymphocyte count remains above 350 cells $/ \mathrm{mm}^{3}$, infections in 
these patients are usually bacterial, including respiratory infections and even tuberculosis ${ }^{2}$. Atypical infections begin to occur as disease progresses and they are characterized by a late response to antibiotic therapy and reactivation of old latent infections ${ }^{3}$. Some opportunistic infections and certain cancers are AIDS-defining conditions, including pneumocystis pneumonia, neurotoxoplasmosis, atypical or disseminated pulmonary tuberculosis, cryptococcal meningitis and cytomegalovirus retinitis ${ }^{4}$. The most common AIDS-related cancers are Kaposi's sarcoma, nonHodgkin's lymphoma and cervical cancer in young women ${ }^{5}$.

Lungs are often the main site of AIDS-related infections and cancers, and patients are treated the same as non-immunodepressed patients. However, it should be remembered that the disease may progress faster in these patients, thus increasing the mortality rate when diseases are undiagnosed or not adequately treated. In addition, the overlaping of more than one illness can make the diagnosis more difficult in some cases. Within this context, fiberoptic bronchoscopy using sampling methods such as bronchoalveolar lavage, bronchial brushing and transbronchial biopsy are of fundamental importance for the integrated work-up of HIV-infected patients ${ }^{6}$. Depending on the clinical suspicion and through the correlation with radiological data, the identification of etiological agents becomes crucial for the adequate treatment and prognosis of these patients ${ }^{7}$.

The aims of this study were to conduct a survey on bronchoscopy procedures performed at the Federal University of Triangulo Mineiro (UFTM) by the Thoracic Surgery Service from 2006 to 2015, to determine the frequency of the main infections diagnosed in patients with HIV/AIDS, to evaluate the diagnostic accuracy of bronchoscopy in these patients, and to correlate the histopathological findings with radiological data and the clinical evolution of the patients.

\section{MATERIALS AND METHODS}

This is a retrospective study conducted at the Special Pathology Unit of the Surgical Pathology Service at the University Hospital of UFTM (HC-UFTM). Bronchoalveolar lavage, bronchial brushing and transbronchial biopsy samples were obtained from HIV-positive and AIDS patients submitted to bronchoscopies performed from 2006 to 2015 and evaluated. HIV-positive serology was confirmed by the Clinical Pathology Service of HC-UFTM or by the Blood Bank of the Regional Blood Center of Uberaba city. Cases with negative serology to HIV were excluded.

Pathologists of the Special Pathology Unit of the Surgical Pathology Service at UFTM, experienced in recognizing these etiological agents in patients with infectious diseases, performed the histopathological study of paraffin-embedded biopsies and cytological specimens (bronchoalveolar lavage and brushing samples). The biopsies underwent hematoxylin and eosin (HE), Grocott and/or periodic acid Schiff staining for the investigation of fungi, and ZiehlNeelsen and/or Fite-Faraco were used for the investigation of mycobacteria. Regarding the cytological specimens, special cytological staining techniques were used for the investigation of microorganisms by HE, Papanicolaou and panoptic staining. The etiological diagnoses were based on the direct microorganisms research, on cytological tests or biopsy results, using the special staining methods mentioned above. No Polymerase Chain Reaction (PCR) techniques or other molecular tests were performed. Clinical and radiological data were retrieved from medical records. The reviewed records were correlated with bronchoscopy results, clinical and radiological findings and the patients' evolution after the start of treatment. All of the data were reviewed by a thoracic surgery specialist. The study was approved by the Ethics Committee of UFTM (Approval N ${ }^{\circ}$ 2.283.777).

\section{RESULTS}

A total of 1,423 patients with a mean age of 50 years were initially selected; 727 cases had no serology for HIV; 696 had serology and 64 were HIV-positive. The cytological specimens and transbronchial biopsies of these patients were reevaluated. The mean age of the group was 47.72 years. Forty-seven $(73.43 \%)$ patients were males, ranging in age from 24 to 84 years (mean 46.13 years). Seventeen $(26.56 \%)$ patients were females, ranging in age from 31 to 69 years (mean 52.12 years).

The transbronchial biopsies and cytological specimens (lavage and/or brushing samples) obtained by fiberoptic bronchoscopy tested positive in 20 (31.25\%) of the 64 HIV-positive patients. Table 1 shows the etiological diagnosis obtained by histopathological and cytological analyses of the samples.

The most frequently diagnosed etiological agent detected by fiberoptic bronchoscopy was Pneumocystis jirovecii (pneumocystis pneumonia), found in 8 of the 64 (12.5\%) HIV-positive patients (Figure 1A and 1B). In all of these cases, the clinical and radiological presentation was compatible with the diagnosis of pneumocystis pneumonia.

The second most common diagnosis was tuberculosis in 4 patients. All of these patients exhibited typical signs and symptoms of the disease, including lymphocytic exudative pleural effusion, pleural thickening, apical lung cavitations, ground-glass appearance and budding-tree images upon radiological examination. 
Table 1 - Histopathological diagnosis by fiberoptic bronchoscopy in HIV-positive patients between 2006 and 2015 at HC-UFTM.

\begin{tabular}{lc}
\hline Histopathological diagnosis & Number of cases (\%) \\
\hline Pneumocystis pneumonia & $8(12.5)$ \\
Tuberculosis & $4(6.25)$ \\
Nocardiosis & $3(4.68)$ \\
Aspergillosis & $1(1.56)$ \\
Cytomegalovirus & $1(1.56)$ \\
Cryptococcosis & $1(1.56)$ \\
Paracoccidioidomycosis & $1(1.56)$ \\
Other mycobacterioses & $1(1.56)$ \\
Negative tests & $44(68.75)$ \\
\hline Total & 64
\end{tabular}

The third most common diagnosis was nocardiosis with visualization of thin, Gram-positive, filamentous bacilli that were isolated from 3 patients (Figure 1C).
Radiological examination revealed homogenous airspace consolidation and areas of bilateral nodular and coalescent infiltration, prone to cavitation, compatible with the diagnostic hypothesis of nocardiosis.

There was one case of each of the following: pulmonary aspergillosis, cytomegalovirus, paracoccidioidomycosis (Figure 1D), atypical mycobacteriosis and cryptococcosis. Although histoplasmosis is a common cause of pulmonary infection in our institution, there were no such cases diagnosed by fiberoptic bronchoscopy in this study.

Disagreement between histopathological and clinical radiological findings was observed in only 2 of the 20 cases in which the fiberoptic bronchoscopy was positive (Table 2), corresponding to $10 \%$ of the positive samples. One case (number 8) was a 37-year-old male patient whose clinical and radiological diagnostic hypotheses were pulmonary tuberculosis, pulmonary abscess and nocardiosis. The transbronchial biopsy revealed a fungal infection caused

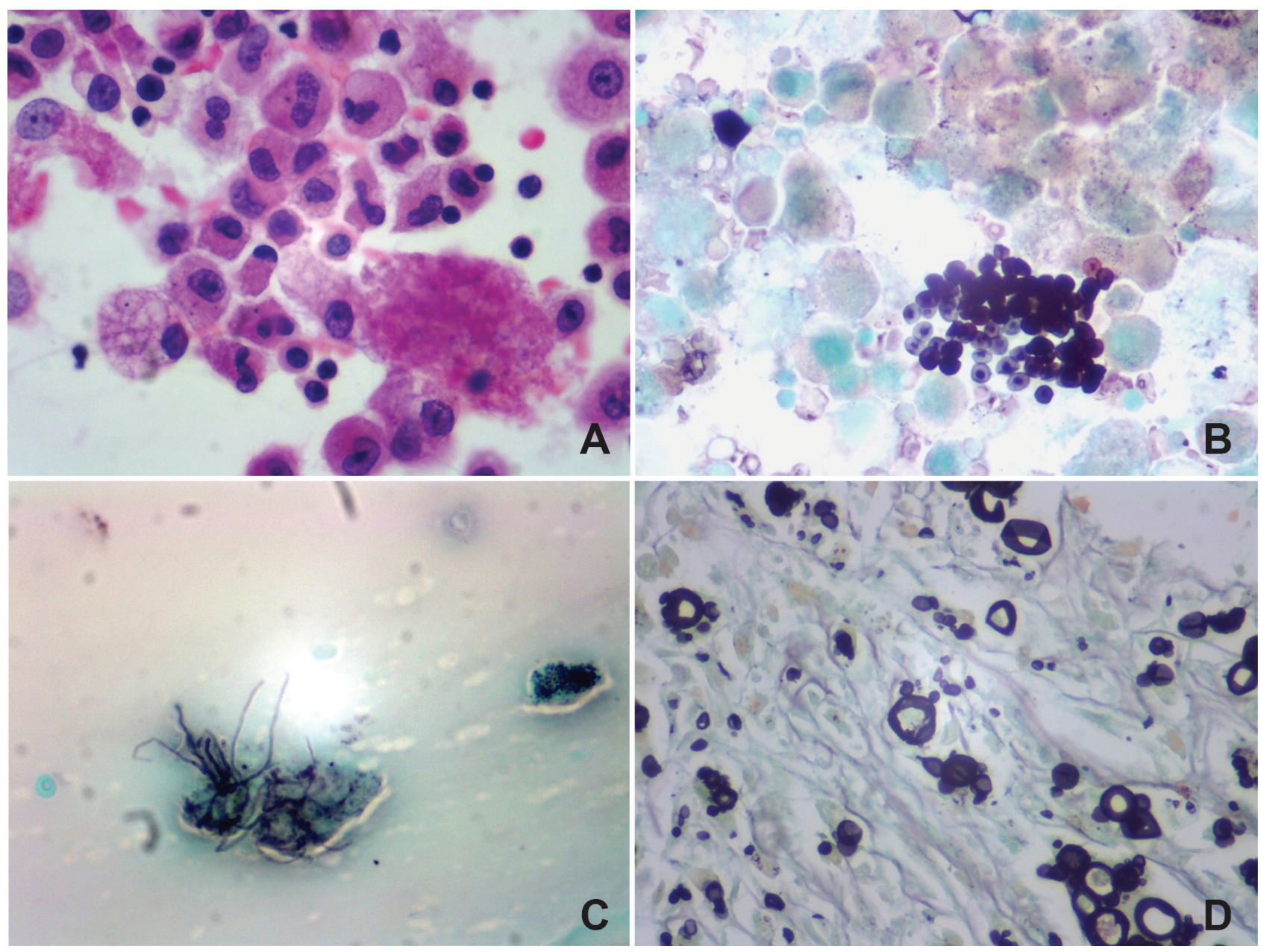

Figure 1 - Photomicrographs of some frequent etiological agents in HIV patients: A) bronchoalveolar lavage fluid showing flocculent and intenseeosinophilic material suggestive of Pneumocystis jiroveci (HE, 400x); B) bronchoalveolar lavage fluid showing rounded, umbilicated and stacked silver-stained structures compatible with Pneumocystis jiroveci (Grocott, 400x); C) bronchoalveolar lavage fluid showing silver-stained filamentous bacteria compatible with Nocardia sp (Grocott, 400x); D) photomicroscopy of lung tissues showing frequent and large spores with multiple and small buds compatible with Paracoccidioides brasiliensis (Grocott, 400x). 
Table 2 - Positive bronchoscopy results in 20 HIV-positive patients according to clinical and radiological findings.

\begin{tabular}{|c|c|c|c|c|c|}
\hline Case & Sex & Age & Bronchoscopy & Clinical finding & Radiology \\
\hline 1 & M & 56 & PCP & PCP & Perihilar opacity \\
\hline 2 & $\mathrm{~F}$ & 51 & Nocardiosis & Nocardiosis & Parenchymatous opacity \\
\hline 3 & M & 60 & PCP & PCP & Parenchymatous opacity \\
\hline 4 & M & 58 & PCP & PCP & Ground-glass opacity \\
\hline 5 & M & 35 & TB & TB & Pleural effusion \\
\hline 6 & M & 38 & PCP & PCP/Pneumonia/TB & Consolidation \\
\hline 7 & M & 45 & PCP & PCP & Ground-glass attenuation \\
\hline 8 & M & 37 & Cryptococcosis & TB/Abscess/Nocardiosis & Abscess/cavern \\
\hline 9 & $\mathrm{~F}$ & 69 & Mycobacteriosis & TB & Opacity/bronchiectasis \\
\hline 10 & M & 44 & PCM & TB/Nocardia/Fungus & Parenchymatous opacity \\
\hline 11 & M & 44 & Nocardiosis & TB/Nocardiosis/Histoplasmosis & No alterations \\
\hline 12 & M & 45 & PCP & PCP & Ground-glass opacity \\
\hline 13 & M & 33 & TB & TB/Fungal infection & Cavitations in apex \\
\hline 14 & M & 50 & PCP & PCP & Nodules/consolidations \\
\hline 15 & M & 59 & TB & TB/Histoplasmosis & Parenchymatous opacity \\
\hline 16 & $\mathrm{~F}$ & 72 & PCP & TB/PCM/PCP/Histoplasmosis & Reduction of soft parts \\
\hline 17 & M & 63 & CMV & TB/KS/Aspergillosis & Hydropneumothorax \\
\hline 18 & M & 84 & Aspergillus & TB/Pneumonia & Opacity/fungal ball \\
\hline 19 & M & 43 & Nocardiosis & Histoplasmosis/Nocardiosis/TB & Parenchymatous opacity \\
\hline 20 & M & 37 & TB & TB/Histoplasmosis & Parenchymatous opacity \\
\hline
\end{tabular}

PCP: pneumocystis pneumonia; TB: tuberculosis; PCM: paracoccidioidomycosis; CMV: cytomegalovirus; KS: Kaposi's sarcoma.

by Cryptococcus neoformans. Liposomal amphotericin treatment was effective in resolving the infection and cure the patient. The other case (case 17) of diagnostic disagreement was a 63-year-old male patient with an extremely compromised immunity. The diagnostic hypotheses were disseminated tuberculosis, Kaposi's sarcoma involving the respiratory system and bronchopulmonary aspergillosis. Involvement of the lung parenchyma accompanied by spontaneous pneumothorax drew attention on imaging studies. Histopathological analysis detected typical viral inclusions of cytomegalovirus. However, in view of the extreme severity of the patient's health conditions, he died before treatment could be started. There was another case (number 18) of an 84-year-old male patient whose histopathological diagnosis was aspergillosis, but there was no clinical suspicion of this infection.and only the radiological examination showed the presence of a fungal ball.

\section{DISCUSSION}

This is a retrospective study that evaluated the bronchoscopy results of bronchoalveolar lavage, brushing and transbronchial biopsy samples in HIV-positive patients attended at HC-UFTM. A total of 696 fiberoptic bronchoscopies were performed at HC-UFTM from 2006 to 2015. Sixty-four of these patients had positive HIV serology and 20 patients (31.25\%) had positive results in the analysis of pulmonary specimens obtained by fiberoptic bronchoscopy. In other study ${ }^{8}$ involving 286 patients with clinical or radiological suspicion of tuberculosis (154 men and 132 women; mean age of 46.7 years old), bronchoscopy contributed to the overall etiological diagnosis in 225 patients (79\%). This percentage is much higher than that obtained in the present study, however, we know that the diagnostic performance and accuracy of bronchoscopy depend on some factors such as adequate sampling avoiding the gross contamination of specimens; the correct distinction between colonization and infection; adequate laboratory processing of samples for histopathological analysis and a satisfactory interaction of the multidisciplinary team involving specialists in infectious diseases, radiologists, surgeons and pathologists. Other studies have reported an even greater diagnostic capacity of bronchoscopy of about $96 \%$ and these authors pointed out the importance of early bronchoscopy to improve its diagnostic efficacy ${ }^{6}$. We do 
not have the exact time of bronchoscopy in these patients, but this examination is usually performed as early as the first days of hospitalization. However, being a tertiary care hospital, sometimes patients arrive at the hospital with weeks or months of disease progression.

Regarding sex and age, most patients were male, in agreement with the literature, and they were slightly older than expected (mean age of 47.72 years in the present study). HIV infection was more common among patients after 30 years of age.

The most frequent finding in the analysis of bronchoalveolar lavage, brushing and transbronchial biopsy samples of HIV-positive patients was pneumocystis pneumonia ( 8 cases, $12.5 \%$ ), followed by tuberculosis (4 cases, $6.25 \%$ ) and nocardiosis ( 3 cases, $4.68 \%$ ). Other authors observed a frequency of tuberculosis of $44 \%$, much higher than the $6.25 \%$ found in our study ${ }^{8}$. It should be noted that the cases in that study were selected based on clinical or radiological suspicions of tuberculosis, while we selected cases according to HIV serology positivity. The bronchoscopic techniques have also contributed to the diagnosis of other conditions such as nonspecific chronic lung inflammation in $51(18 \%)$ patients, pneumocystis pneumonia, fungal infections or nocardiosis in 20 (7\%) patients, bronchiolitis obliterans organizing pneumonia, alveolitis and pneumoconiosis in $14(5 \%)$ patients, pulmonary or metastatic tumors in 7 (2\%) patients, and non-tuberculous mycobacteriosis in $6(2 \%)^{8}$ patients. The frequency of pneumocystis pneumonia, nocardiosis and fungal infections was much higher in our study, totaling $21.86 \%$, compared to that reported by others ${ }^{8}$ for the same reason, i.e., we selected HIV-immunosuppressed patients who are known to have a higher frequency of these opportunistic infections. In a study carried out in a referral hospital in Lima, Peru ${ }^{7}$, the authors have also found a higher frequency of tuberculosis (23\%) in the studied bronchoalveolar lavage samples. The patients of that study were also selected based on clinical suspicions of tuberculosis. Another important point is the technique used for the diagnosis. We performed the direct search of microorganisms in liquid or tissue samples through special staining methods such as Fite Faraco and Ziehl-Neelsen; other authors used culture and/or PCR techniques to search for etiological agents, thus increasing the chances of reaching a diagnosis ${ }^{7,8}$.

In another study conducted in Africa ${ }^{9}$ on HIV-positive patients undergoing fiberoptic bronchoscopy, the authors observed a $23 \%$ frequency of tuberculosis, $13 \%$ of cryptococcosis, $9 \%$ of Kaposi's sarcoma, $5 \%$ of pneumocystis pneumonia and $38 \%$ of nonspecific interstitial pneumonias. They commented on the lower frequency of pneumocystis pneumonia in Africa and indicated tuberculosis as the most common cause of pulmonary involvement in HIV-positive patients. On the other hand, pneumocystis pneumonia was the most common finding in the HIV-positive patients in the present study, a fact that has also been observed in North America and Europe.

Disagreement between the diagnosis established by histopathological analysis and the clinical radiological findings was observed in only 2 of the 20 cases in which fiberoptic bronchoscopy was positive, corresponding to $10 \%$ of the sample diagnosed by bronchoscopy. Hence, the sensitivity and specificity of bronchoscopy were $31.25 \%$ and $90 \%$, respectively, for the diagnosis of pulmonary affections in HIV-positive patients by using bronchoalveolar lavage, brushing and transbronchial biopsy. Jacomelli et al. ${ }^{8}$ found sensitivity and specificity of bronchoalveolar lavage of $60 \%$ and $100 \%$, respectively, for the specific diagnosis of tuberculosis in patients with clinical and/or radiological suspicion. A considerable increase in sensitivity to $84 \%$ was observed when the procedure was combined with transbronchial biopsy.

Fiberoptic bronchoscopy remains a common procedure for assessing pulmonary disorders at HC-UFTM, especially in HIV-positive patients. Analysis of cytological and biopsied specimens obtained by fiberoptic bronchoscopy is a valuable tool for theetiological diagnosis of pulmonary infections in HIV-positive patients.

When positive, samples of bronchoalveolar lavage, brushing and transbronchial biopsy confirm the hypotheses raised, but negative results do not always exclude the disease, so clinical and imaging examinations are fundamental to the choice of treatment.

\section{ACKNOWLEDGMENTS}

To the medical colleagues of the Surgical Pathology Unit for the biopsies and cytological specimens and to the Clinical Pathology Service for performing serological tests.

\section{AUTHORS' CONTRIBUTIONS}

JPVS: review of cases and preparation of the text; LFGL: review of cases and preparation of the text; SJA: performed biopsies and cytological tests and review of the text; MLSV: review of clinical data and of the text; AMRM: performed the biopsies and cytological tests review of cases and preparation of the text.

\section{REFERENCES}

1. Brasil. Ministério da Saúde. Secretaria de Atenção à Saúde. Departamento de DST, AIDS e Hepatites virais. Protocolo 
clínico e diretrizes terapêuticas para prevenção da transmissão vertical de HIV, sífilis e hepatites virais. Brasília: Ministério da Saúde; 2015.

2. Brasil. Ministério da Saúde. Secretaria de Vigilância em Saúde. Programa Nacional de DTS e AIDS. Manual de rotinas para assistência de adolescentes vivendo com HIV/AIDS. Brasília: Ministério da Saúde; 2006.

3. Bezabhe WM, Chalmers L, Bereznicki LR, Peterson GM. Adherence to antirretroviral therapy and virologic failure: a meta-analysis. Medicine (Baltimore). 2016; 95:e3361.

4. Boyton RJ, Altmann DM, Wright A, Kon OM. Pulmonary infection with Cryptococcus neoformans in the face of underlying sarcoidosis. Respiration. 2007;74:462-6.

5. Cadranel J, Naccache J, Wislez M, Mayaud C. Pulmonary malignancies in the immunocompromised patient. Respiration. 1999;66:289-309.
6. Broaddus C, Dake MD, Stulbarg MS, Blumenfeld W, Hadley WK, Golden JA, et al. Bronchoalveolar lavage and transbronchial biopsy for the diagnosis of pulmonary infections in the acquired immunodeficiency syndrome. Ann Intern Med. 1985;102:747-52.

7. Soto A, Salazar D, Acurio V, Segura P, Van der Stuyft P. Evaluation of the diagnostic utility of fiberoptic bronchoscopy for smearnegative pulmonar tuberculosis in routine clinical practice. J Bras Pneumol. 2012;38:757-60.

8. Jacomelli M, Silva PR, Rodrigues AJ, Demarzo SE, Seicento M, Figueiredo VR. Bronchoscopy for the diagnosis of pulmonary tuberculosis in patients with negative sputum smear microscopy results. J Bras Pneumol. 2012;38:167-73.

9. Batungwanayo J, Taelman H, Lucas S, Bogaerts J, Alard D, Kagame A, et al. Pulmonary disease associated with the human immunodeficiency virus in Kigali, Rwanda. A fiberoptic bronchoscopy study of 111 cases of undetermined etiology. Am J Respir Crit Care Med. 1994;149:1591-6. 